\title{
Aggregation in mixing tanks - the role of inter-particle forces
}

\author{
Jee Wen Lim \& J.J. Derksen \\ School of Engineering, University of Aberdeen, Aberdeen, UK \\ jderksen@abdn.ac.uk \\ Submitted to Chemical Engineering Research \& Design - April 2019 \\ Revision submitted: July 2019 \\ $2^{\text {nd }}$ revision submitted: August 2019
}

Accepted: August 2019

\begin{abstract}
We study aggregation of equally-sized spherical particles under mildly turbulent flow conditions (Reynolds numbers in the range 4000 to 8000 ) in a mixing tank through numerical simulation. The dynamics of the liquid flow is solved in terms of the volume-averaged Navier-Stokes equations by an extended lattice-Boltzmann method on a fixed uniform cubic grid. The particle dynamics is updated through applying Newton's second law to each particle. The simulations include a two-parameter model for the attractive force between the particles that causes aggregation. The dynamics of solids and liquid are two-way coupled through a mapping procedure. An overall solids volume fraction of $10 \%$ has been investigated. The level of aggregation of particles in the mixing tank mainly depends on the strength of the attractive force and on the impeller-based Reynolds number, not so much on the distance over which the aggregative force is active. A higher Reynolds number leads to less aggregation.
\end{abstract}

\section{Keywords}

Solids suspension, turbulent flow, lattice-Boltzmann method, aggregation, two-way coupling, EulerianLagrangian simulation 


\section{Introduction}

In the process industries, solid-liquid mixing is applied to enhance mass transfer between a solids phase and a liquid by exposing as much as possible interfacial area to fluid flow. Examples are dissolution or particle formation processes as they take place in industrial crystallization [Mesbah et al 2009; Nagy et al 2013]. Often in such systems, particles have a tendency to aggregate. This can be a desired phenomenon if the purpose is to quickly grow particles and separate them from the liquid through filtering or by letting them settle as in coagulation/flocculation steps in water treatment [Bache \& Gregory, 2007; Dionisi 2017]. Aggregation can be unwanted, representing an operational problem, as it reduces interfacial area or deteriorates product quality as it could result in an undesirably wide distribution of particle sizes [Tourbin \& Frances 2009].

In order for aggregation to happen, particles need to collide. In agitated systems, particles mainly collide due to velocity differences induced by fluid flow, with collision frequencies being a strong function of solids volume fraction. The fraction of collisions that results in particles aggregating is known as the collision efficiency [Elimelech et al 2013; Njobuenwu \& Fairweather 2017]. In mixing tanks, aggregation of particles and breakage of aggregates occur simultaneously. Breakage of aggregates can be caused by collisions with other particles or aggregates, collisions with the impeller or tank walls, and as a result of forces associated to fluid deformation [Seto et al 2011]. Factors influencing breakage of aggregates include structure, size and strength of aggregates. As an example, if we compare a compact aggregate with an aggregate having an open structure made up of the same number of primary particles, the latter breaks more easily because the fluid velocity differences over the aggregate are larger leading to stronger fluid deformation forces on the aggregate [Horii et al 2015], as well as because open aggregates are weaker since the number of contact points per primary particle is less. A detailed quantitative picture of aggregation benefits from studying the interactions between solids and liquid as well as between particles during fluid flow and mixing [Tamburini et al., 2012; Tamburini et al., 2014; Qi et al., 2013; Mishra \& Ein-Mozaffari, 2017; Blais et al., 2017]. In the current paper, these interactions are studied 
based on computational fluid dynamics (CFD). Several numerical methods have been developed for simulating particle aggregation under turbulent flow conditions [Reeks 2014; Njobuenwu \& Fairweather 2017; Njobuenwu \& Fairweather 2018]. Previous studies have observed that the level of aggregation, aggregate sizes, and the distribution of aggregates over the flow domain are intimately related to hydrodynamic conditions [Clark \& Flora 1991; Spicer et al 1998; Kobayashi et al 1999; Kobayashi 2004; Tourbin \& Frances 2009].

The aim of this paper is to quantify - by means of numerical simulations - the aggregation process as it takes place in a mixing tank as a function of flow conditions and particle properties, the latter including the characteristics of the forces causing aggregation. The aggregation of particles is quantified in terms of coordination numbers, i.e. the number of particles a reference particle is in contact with, aggregate size distributions, and the solids volume fraction distribution over the mixing tank volume. Every particle is of the same size and is equipped with a force that attracts other particles and causes them to aggregate. The force has two parameters: the strength of the force and the distance over which the attraction acts.

We deal with a generic, aggregating system that we characterize through a set of dimensionless parameters. The flow induced by the impeller is characterized by a Reynolds number, a Shields number expresses the competition of particle suspension by flow and settling by net gravity, the solids volume fraction and the solids over liquid density ratio further characterize the particulate system. The two parameters of the aggregation force model (strength and distance) have been non-dimensionalized by the gravity force acting on a particle and the particle size respectively. In this paper, the dimensionless parameters that have been varied independently to study their impact on the levels of aggregation are the Reynolds number and the aggregation force parameters. Other parameters have been fixed to constant values.

In this paper we simulate the solids-liquid flow based on an Eulerian-Lagrangian approach: a fixed, three-dimensional grid of nodes is applied for calculating the flow of the continuous liquid phase 
(Eulerian) while the discrete phase (particles) is tracked in a Lagrangian manner [Capecelatro \& Desjardins 2013; Blais et al., 2016]. In this paper, the Eulerian-Lagrangian approach using particleunresolved simulations has been adopted. Particles that are of comparable size to the grid spacing [Derksen 2018a] are tracked individually by solving Newton's second law of motion that includes hydrodynamics forces, aggregation forces and contact forces. The information exchange between particles (Lagrangian) and the liquid (Eulerian) is achieved by mapping functions: Lagrangian quantities are distributed over the grid and weighted averages of Eulerian quantities are assigned to particles based on their center locations relative to the grid [Deen et al 2004; Capecelatro \& Desjardins 2013; Derksen 2018a].

This paper is organized as follows: First, the flow system is introduced. We then briefly summarize the simulation procedure covering the numerical methods of the solids and liquid phases, together with the methods implemented to handle particle-particle interactions and aggregation and lastly the coupling of both phases. In the following section, results regarding the aggregation of particles in agitated suspensions are presented and discussed. Finally, we draw conclusions on the outcome of our study and provide perspectives for further study.

\section{Flow system}

Figure 1 shows the mixing tank and defines the coordinate system. The tank is cubic with side length $T$ and is closed off by a lid so that no-slip conditions apply on all six outer boundary planes. The impeller placed in the center of the tank with an off-bottom clearance of $C=T / 3$ - has four $45^{\circ}$ pitched blades that pump the liquid downwards, i.e. in the negative $z$-direction. It has angular velocity $N$ (rotations per unit time). The tank contains a Newtonian fluid with density $\rho$ and kinematic viscosity $\nu$, and equally sized spherical solid particles of diameter $d$ and density $\rho_{s}, \rho_{s}>\rho$. Gravity points in the negative $z-$ direction: $\mathbf{g}=-g \mathbf{e}_{\mathbf{z}}$. 
The particles tend to aggregate as a result of an attractive force acting in the radial direction. The properties of the aggregative force are discussed here rather than in the next section (which is mostly on numerics) since the force is part of the physical flow system we study. It also is part of the nondimensional representation of the simulations, as discussed later in this section. The aggregative force on particle $i$ due to a nearby particle $j$ is

$$
\mathbf{F}_{\mathbf{i j}}=\beta \frac{\left(\delta_{0}-\delta\right)}{\delta_{0}} \frac{\left(\mathbf{x}_{\mathbf{j}}-\mathbf{x}_{\mathbf{i}}\right)}{\left|\mathbf{x}_{\mathbf{j}}-\mathbf{x}_{\mathbf{i}}\right|} \text { if } 0<\delta<\delta_{0} ; \mathbf{F}_{\mathbf{i j}}=\beta \frac{\left(\mathbf{x}_{\mathbf{j}}-\mathbf{x}_{\mathbf{i}}\right)}{\left|\mathbf{x}_{\mathbf{j}}-\mathbf{x}_{\mathbf{i}}\right|} \text { if } \delta \leq 0 ; \mathbf{F}_{\mathbf{i j}}=\mathbf{0} \text { otherwise }
$$

where $\delta=\left|\mathbf{x}_{\mathbf{j}}-\mathbf{x}_{\mathbf{i}}\right|-d$ the distance between the two spherical particle surfaces, $\beta$ the force when the particles are in contact (i.e. when $\delta \leq 0)$ and $\delta_{0}$ the distance below which the force becomes active. The magnitude of the force thus linearly increases from 0 at $\delta=\delta_{0}$ to $\beta$ at $\delta=0$. It saturates if the particles overlap $(\delta<0)$. The total aggregative force particle $i$ experiences is the sum of forces due to all its surrounding particles: $\mathbf{F}_{\mathbf{i}}=\sum_{j \neq i} \mathbf{F}_{\mathrm{ij}}$.

We will be using a set of dimensionless numbers to characterize the system sketched above. The flow geometry gives rise to a number of aspect ratios for which the side length of the (cubic) $\operatorname{tank} T$ has been taken as the reference length. These aspect ratios are fixed in this paper. The most important ones relate to the impeller diameter: $D / T=0.44$, and the distance between the impeller and the tank bottom: $C / T=\frac{1}{3}$. The liquid flow in the tank is characterized by an impeller-based Reynolds number $\operatorname{Re}=N D^{2} / \nu$. The inclusion of the aggregative solids phase comes with the following dimensionless numbers: (1) the solids volume fraction $\langle\phi\rangle=n \frac{\pi}{6} d^{3} / T^{3}$ with $n$ the number of particles in the tank; (2) the particle size relative to the tank size $d / T$; (3) the density ratio $\rho_{s} / \rho$; (4) a modified Shields number [Derksen 2012] that quantifies the ratio of liquid inertial stress and net gravity on the particles $\theta=\frac{\rho N^{2} D^{2}}{g d\left(\rho_{s}-\rho\right)} ;$ (5) the strength of the aggregation force relative to the weight of a particle 
$\beta_{a}=\beta /\left(g \rho_{s} \frac{\pi}{6} d^{3}\right) ;$ and (6) the distance over which the aggregative force is active relative to the particle size $\delta_{a}=\delta_{0} / d$.

As an alternative for normalizing $\beta$ with the particle weight, it also could be normalized with a measure for the inertial hydrodynamic force on a particle: $\frac{\beta}{\rho N^{2} D^{2} d^{2}}$. We note that the latter dimensionless group is a combination $\beta_{a}$, the Shields number $\theta$ and the density ratio: $\frac{\beta}{\rho N^{2} D^{2} d^{2}}=\frac{6}{\pi} \frac{\beta_{a}}{\theta\left(\rho_{s} / \rho-1\right)}$

As can be concluded, this is a multi-dimensional parameter space of which only a small part has been covered. This paper primarily looks into the impact the parameters $\beta_{a}$ and $\delta_{a}$ on the levels of aggregation. Also the Reynolds number has been varied; between 4,000 and 8,000. That range indicates transitional to mildly turbulent flow [Zhang et al 2017]. This choice was made mainly for computational reasons. It allows us - at this stage of our research - to perform the liquid flow simulations without the use of a turbulence model so that we do not need (speculative) models for solid particle motion due to unresolved turbulent fluctuations. The other dimensionless numbers have been given fixed values in this paper. Table 1 shows these values (except for the stirred tank geometrical aspect ratios that are given in Figure 1) as well as the ranges covered by $\operatorname{Re}, \beta_{a}$ and $\delta_{a}$. With a reference to Table 1 , we identify the conditions with $\mathrm{Re}=4,000$ as base-case conditions.

\section{Numerical procedures}

Most of the details of the numerical procedures as applied in this paper have been published in two recent papers [Derksen 2018a; Derksen 2018b]. A short summary, highlighting the major assumptions and modeling steps, is given here. In addition, it will be discussed how the aggregative force has been implemented. 
The liquid dynamics satisfies the volume-averaged continuity and Navier-Stokes equations [Sankaranarayanan \& Sundaresan 2008]:

$$
\begin{gathered}
\frac{\partial}{\partial t}\left(\rho \phi^{c}\right)+\nabla \cdot\left(\rho \phi^{c} \mathbf{u}\right)=0 \\
\frac{\partial}{\partial t}\left(\rho \phi^{c} \mathbf{u}\right)+\nabla \cdot\left(\rho \phi^{c} \mathbf{u u}\right)=\phi^{c} \nabla \cdot \boldsymbol{\pi}+\mathbf{f}_{\mathrm{s}}
\end{gathered}
$$

with $\phi^{c} \equiv 1-\phi$ the liquid volume fraction and $\phi$ the solids volume fraction, $\mathbf{u}$ the interstitial liquid velocity, $\boldsymbol{\pi}$ the liquid's stress tensor, and $\mathbf{f}_{\mathrm{s}}$ the force per unit volume the solid particles exert on the liquid. We solve Eqs. 2 \& 3 in a three-dimensional, time-dependent manner. The solids volume fraction and therefore $\phi^{c}$ (including its temporal and spatial derivatives) is available from the locations of the solid particles. The latter are calculated by integrating the equations of motion of each individual particle. The forces included in the equations of motion are the drag force due to slip velocity between liquid and solid, net gravity, a soft-sphere contact force that takes care of collisions between particles and between particles and the impeller and tank walls, a radial lubrications force that models short-range hydrodynamic interactions [Derksen 2018b], and the aggregative force the characteristics of which have been discussed above.

The drag force is written in the following form: $\mathbf{F}_{\mathbf{D}}=3 \pi \rho v d\left(\mathbf{u}-\mathbf{u}_{\mathbf{p}}\right) F\left(\operatorname{Re}_{p}, \phi\right)$ with $\mathbf{u}_{\mathbf{p}}$ the particle velocity and $F$ the drag coefficient that is a function of a particle-based Reynolds number $\operatorname{Re}_{p}=(1-\phi)\left|\mathbf{u}-\mathbf{u}_{\mathbf{p}}\right| d / v$ as well as of the local solids volume fraction $\phi$. In the current study, $F\left(\operatorname{Re}_{p}, \phi\right)=\left(1+0.15 \operatorname{Re}_{p}^{0.687}\right)(1-\phi)^{-2.65}$. The Reynolds number dependency is due to Schiller \& Naumann [Schiller \& Naumann 1933] while the solids volume fraction dependency is due to Wen \& Yu [Wen \& $\mathrm{Yu}$ 1966]. It has been demonstrated that the Wen \& Yu relationship is most appropriate for moderate Stokes number systems as we have here with solid particles in liquid [Rubinstein et al 2016] with a density ratio of order 1 . The drag force on the particles is fed back to the liquid and enters the volumeaveraged Navier-Stokes equations in the form of the body force $\mathbf{f}_{\mathrm{s}}$. 
For the details regarding the mathematical expressions for the contact and lubrication forces that exist between nearby particles we refer to our earlier work [Derksen 2018a]. Evaluating these forces requires identifying nearby particles for which we have an efficient link-list algorithm. This we also use to evaluate the aggregative force that solely depends on the distance between particles in close proximity, see Eq. 1.

An important aspect of the simulations is the exchange of information between the fluid flow and the solid particle dynamics. The fluid flow is represented by velocity and pressure values on an (Eulerian) grid of points; the particles by their properties at their center locations (Lagrangian). A three-dimensional mapping function has been used to distribute particle properties over the grid, and to determine fluid properties at the center locations of the particles. An example of the former is the determination of the Eulerian solids volume fraction field $\phi$ (as it appears in Eqs. $2 \& 3$ ) from the particle center locations; an example of the latter is the determination of the liquid velocity in the direct vicinity of a particle to evaluate the drag force. As the mapping function we have been using a clipped fourth-order polynomial [Deen et al 2004]. In one spatial dimension $\xi$ it reads

$$
\mu(\xi)=\frac{15}{16}\left[\frac{\xi^{4}}{\lambda^{5}}-2 \frac{\xi^{2}}{\lambda^{3}}+\frac{1}{\lambda}\right] \text { for }-\lambda \leq \xi \leq \lambda ; \quad \mu(\xi)=0 \text { for }|\xi|>\lambda
$$

with $\lambda$ the half-width of the mapping function. In three dimensions it is the product $\mu\left(\xi_{1}\right) \mu\left(\xi_{2}\right) \mu\left(\xi_{3}\right)$ with $\xi_{i} i=1 \ldots 3$ three Cartesian coordinates. As in previous papers [Derksen 2018a; Derksen 2018b], based on benchmarking with particle-resolved simulations [Derksen 2018a], we have set $\lambda=1.5 d$.

The volume averaged Navier-Stokes equations have been solved by an extended lattice-Boltzmann (LB) scheme. Full details can be found in [Sungkorn \& Derksen 2012]. The scheme operates on a uniform cubic lattice with spacing $\Delta$ and takes time steps $\Delta t$. The single-phase variant [Eggels \& Somers 1995] is an early version of a multiple relaxation time (MRT) scheme with different moments being relaxed at different rates. The multiphase extension involves mass and momentum sources in order to deal with fluid mass being displaced by solids mass (and vice versa). 
An immersed boundary method has been used to impose the no-slip conditions at the surfaces of the impeller that drives the liquid flow [Derksen \& Van den Akker 1999]. The no-slip condition at the bounding (tank) walls has been imposed through half-way bounce back of the LB distribution functions [Succi 2001]. The equations of motion of the particles have been updated with a split-derivative scheme [Feng \& Michaelides 2009] that takes time steps of the same size as the lattice-Boltzmann scheme.

\section{Set-up of simulations}

The tank volume has been discretized by a uniform, cubic grid of $110^{3}$ nodes with spacing $\Delta$. In this tank volume we place 250,000 non-overlapping spheres with diameter $d=\Delta$ and so achieve a solids volume fraction of $\langle\phi\rangle=0.0983$. These particles we let settle under gravity where they eventually form a loosely packed layer of solids with a thickness of approximately $20 d$ on the bottom of the tank. We then set fluid and particle velocity explicitly equal to zero and start rotating the impeller. The impeller makes one revolutions in $1600 \Delta t$. With an impeller diameter of $D=48 \Delta$, the tip speed of the impeller then is $\mathrm{v}_{t i p}=0.094 \Delta / \Delta t$. The tip speed is a good measure for the maximum liquid speed in the tank. Its value being an order of magnitude smaller than $\Delta / \Delta t$ indicates that we solve a nearly incompressible flow with the - by its nature - compressible LB scheme [Succi 2001].

Grid effects have been studied extensively in a previous paper involving non-aggregating particles [Derksen 2018b]. Based on the results presented there we acknowledge that the currently used grid - that spans 110 grid spacings $\Delta$ in each coordinate direction - is a compromise between accuracy and computational affordability. The results for $\mathrm{Re}=8,000$ (the highest impeller-based Re number used) are somewhat under-resolved. The latter can be appreciated by estimating the Kolmogorov length scale as $\eta=T \mathrm{Re}^{-3 / 4}$. With $\mathrm{Re}=8,000$ and $T=110 \Delta, \eta=0.13 \Delta$. A requirement for well resolved DNS of turbulence [Moin \& Mahesh 1998] is $\Delta \leq \pi \eta$ which would thus ask for a 2.4 times finer grid in each direction. This is in line with results in [Derksen 2018b] that indicate grid independence starting from grids with $T=220 \Delta \quad($ at $\operatorname{Re}=4,000)$. 


\section{Results}

We first created a non-aggregating suspension $\left(\beta_{a}=0\right)$ at $\operatorname{Re}=4000$ and further the conditions as defined in Table 1. Starting from the initial condition with the particles forming a dense solids layer on the bottom of the tank we simulate 100 impeller revolutions $(t N=100)$. The way the system evolves has been visualized in Figure 2 in terms of particle locations and in Figure 3 in terms of the liquid velocity field in a vertical plane through the center of the tank. Once the flow generated by the impeller reaches the particle bed (after a few impeller revolutions) it starts suspending particles. At these initial stages, we observe a strong coupling between fluid flow and particles. When the particles are not fully suspended, they clearly obstruct the fluid flow in the bottom region of the tank. At the end of this simulation, at $t N=100$, the fluid flow has reached the upper regions of the tank and particles are distributed over the entire tank volume. The liquid stream coming off the impeller is still somewhat deflected horizontally due to the high particle concentration in the bottom region. To quantify the particle distribution, Figure 4 shows the time-averaged solids volume fraction distribution in a vertical cross section. As can be seen, the distribution is far from uniform with regions near the bottom and along the side walls having elevated levels of solids.

An important parameter that has been used to quantify aggregation is the coordination number. If associated with an individual particle, it is the number of other particles that individual particle is in contact with. "Contact" (between particle $i$ and $j$ ) in this respect is defined as $\delta=\left|\mathbf{x}_{\mathbf{j}}-\mathbf{x}_{\mathbf{i}}\right|-d \leq 0$, i.e. the particles touch or overlap. Averaging over all particles in the mixing tank results in the average coordination number, symbol $C_{a v g}$. The way it evolves in time for the non-aggregating system is shown in Figure 5. The level of $C_{\text {avg }} \approx 5$ the time series starts with is the result of particles resting on one another in the bottom solids layer that serves as the initial condition. Over a period of approximately 50 
impeller revolution, $C_{a v g}$ reaches a steady state of approximately 0.5 . Particles are thus in regular contact with other particles, also without the aggregation force having been activated.

The instantaneous state of the system (the liquid as well as the solids dynamics) at the end of the non-aggregation simulation (at $t N=100$ ) has been used as the starting point for simulations with activated aggregation forces. At a fixed dimensionless aggregation distance of $\delta_{a}=0.4$, the interaction strength was varied in the range $0.1 \leq \beta_{a} \leq 27$. Time series of $C_{a v g}$ are given in Figure 6 (where we have reset the clock to $t N=0$ at the start of the aggregating simulations). The average coordination number responds strongly and quickly to switching on the aggregation force. Even with $\beta_{a}=1$ there is a clear effect; for $\beta_{a}=0.1$ the aggregative force has virtually no effect on $C_{a v g}$. The time scales over which the solid-liquid flow evolves to a new steady state are in the range of 10 to (at least) 60 impeller revolutions. Qualitative impressions of the evolution of aggregation for the case with $\beta_{a}=9$ are given in Figure 7. It is interesting to note here that after 16 impeller revolutions the aggregated solids avoid the region around the impeller where the liquid flow is strongest. The particles that are in that region are single or part of small aggregates.

With increasing aggregation strength $\beta_{a}$, the solids distribution over the tank volume gets more and more inhomogeneous, see Figure 8 that shows time-averaged contours of the solids volume fraction $\phi$ in a vertical plane after a dynamic steady state has been reached. Where for $\beta_{a}=1$ the $\phi$ distribution is much in line with that for the non-aggregating system (Figure 4), for higher $\beta_{a}$ the particles concentrate near the bottom and - to a lesser extent - the top of the tank and avoid the regions with strong, impellerinduced flow. Flocs collecting near the bottom it is to be expected given the increased settling speed of aggregates compared to single particles. A high concentration of solids in the upper parts of the tank is somewhat surprising. Aggregation there is relatively strong there because there is only weak flow so that aggregates can grow large before (sometimes) broken by liquid deformation. 
Remarkably, the interaction distance $\delta_{a}$ has very limited effect on the levels of aggregation as measured by $C_{a v g}$. In Figure 9 we show steady state $C_{a v g}$ values as a function of $\beta_{a}$. For a number of $\beta_{a}$ values we have three settings of the interaction distance. Increasing $\delta_{a}$ from 0.3 to 0.5 increases $C_{a v g}$ at most by $8 \%$ (when $\beta_{a}=3$ ).

We have studied the effect the Reynolds number has on the aggregation process. In this simulation study we did this by changing the kinematic viscosity of the fluid. This then only changes the Reynolds number; the other dimensionless numbers are independent of $\nu$ (see Table 1). This would be different from an experiment where the natural way of changing the Reynolds number is changing the impeller speed which, however, also changes the Shields number $\theta$ and thus the level of suspension by modifying the balance between flow (inertial stress) and net gravity. For an aggregative force with $\beta_{a}=3$ and $\delta_{a}=0.4$, the Reynolds number has a clear effect on aggregation levels as measured by the average coordination number with the steady values of $C_{\text {avg }}$ decreasing with increasing Re, see Figure 10 . The stronger flow and - more specifically - the stronger turbulence at higher Reynolds numbers shifts the equilibrium between breakage and aggregation slightly towards more breakage. Evidence for stronger turbulence at higher Re is provided in Figure 11 where time average contours of normalized turbulent kinetic energy are plotted for the three Reynolds numbers considered, viz. $\mathrm{Re}=4000,6000$ and 8000 . Given that at $\mathrm{Re}=8000$ we under-resolve the flow, the Re-trends observed might actually be stronger. We leave this for future work.

Next to coordination numbers, levels of aggregation can be characterized in terms of aggregate size distributions. An aggregate is defined as a set of connected primary particles where "connected" is defined in the same way as it was for determining the coordination number: $\delta \leq 0$. For low aggregative strength $\left(\beta_{a} \leq 1\right)$ this leads to sensible aggregate size distributions, see Figure 12a. The fraction of single particles decreases with increasing $\beta_{a}$, and more and larger aggregates appear. 
Starting from $\beta_{a}=3$, however, we see instances where very large "aggregates" form and - in the next instance - disappear. This leads to aggregate size distributions as shown in Figure $12 \mathrm{~b}$ with aggregates containing of the order of $10^{5}$ particles, i.e. approximately $50 \%$ of the total number of particles in the tank. The reason for their existence is that we are dealing with fully reversible aggregation \& breakage and with a dense (solids volume fraction of 10\%) suspension so that particles connect over distances of the order of the tank size. That these huge "aggregates" make up only a small fraction $\bar{\psi}$ is because they are very short lived and the distribution in Figure $12 \mathrm{~b}$ is an average over much longer time (10 impeller revolutions). These large aggregates cannot be observed experimentally; e.g. extracting a sample from the mixing tank will inevitable break them. We conclude that characterizing the levels of aggregation in terms of an aggregate size distribution in the type of simulations we do requires careful interpretation.

\section{Conclusions}

This paper reports results of simulations of solid-liquid mixing with particles that have a tendency to aggregate. They Eulerian/Lagrangian approach involves two-way coupling between solid and liquid, as well as a direct treatment of collisions between particles based on a repulsive contact force. These are necessary features since the solids volume fraction is at a moderately high level (10\%). The liquid flow is mildly turbulent and we do not apply a turbulence or subgrid-scale stress model. A simple, twoparameter, reversible aggregative force model has been devised; one parameter characterizes the distance of interaction, the other the strength of interaction.

The simulations provide detailed insights in how the flow interacts with aggregation and break-up processes. We observe, for instance, aggregation taking place away from the strong flow induced by the impeller, and related to this an effect of the Reynolds number on the levels of aggregation. 
The aggregation process has been characterized by keeping track of the evolution of the coordination number averaged over all particles in the mixing tank. Levels of aggregation strongly depend on the aggregation strength. The distance over which the aggregative force acts has minor effect.

Weak aggregative forces generate reasonable aggregate size distributions; strong forces lead to particles connecting over distances of the order of the container size. This we consider an artifact of the relatively simple and reversible - force model, or of the way we define an aggregate. This is a topic for further research. For this we will be looking into more realistic force models involving, for instance, the contact time between particles during which the particles grow a bond. Such more complex - and likely irreversible - force models will have more than two model parameters so that we are looking for specific (real) solid-liquid systems and experimental data to guide the choices for the model parameters.

The design of experiments to support validation of our numerical procedure is very intricate. Measuring aggregate size distributions (preferably in a local manner) by taking samples will suffer from invasiveness and aggregates breaking or merging in the sampling process. A better option might be to visualize the solids distribution in the tank, preferably by near-refractive-index-matching of liquid and solids so that we can look deep in the tank. Figure 8 shows profound effects of the strength of aggregation on the solids distribution over the tank volume.

Future work also involves flocculation type computational experiments. We plan on adding a limited number of "active" aggregative particles to a system containing non-aggregating particles and study to what extent mixing-in these active particles can flocculate the passive particles. 


\section{References}

Bache, D. H., \& Gregory, R. (2007). Flocs in water treatment. IWA publishing, London.

Blais, B., Lassaigne, M., Goniva, C., Fradette, L. \& Bertrand, F. (2016). Development of an unresolved CFD-DEM model for the flow of viscous suspensions and its application to solid-liquid mixing, Journal of Computational Physics, 318, 201-221.

Blais, B., Bertrand, O., Fradette, L. \& Bertrand F. (2017). CFD-DEM simulations of early turbulent solidliquid mixing: Prediction of suspension curve and just-suspended speed, Chemical Engineering Research and Design 123, 388-406.

Capecelatro, J. \&, Desjardins, O. (2013). An Euler-Lagrange strategy for simulating particle-laden flow, Journal of Computational Physics, 238, 1-31.

Clark, M. M., \& Flora, J. R. (1991). Floc restructuring in varied turbulent mixing. Journal of Colloid and Interface Science, 147, 407-421.

Deen, N. G., van Sint Annaland, M., \& Kuipers, J. (2004). Multi-scale modeling of dispersed gas-liquid two-phase flow. Chemical Engineering Science, 59, 1853-1861.

Derksen, J. J. \& Van den Akker, H. E. A. (1999). Large eddy simulations on the fl ow driven by a Rushton turbine, AIChE Journal, 45, 209-221.

Derksen, J.J. (2012). Highly resolved simulations of solids suspension in a small mixing tank. AIChE Journal, 58, 3266-3278.

Derksen, J.J. (2018a). Assessing Eulerian-Lagrangian simulations of dense solid-liquid suspensions settling under gravity. Computers \& Fluids, 176, 266-275.

Derksen, J.J. (2018b). Eulerian-Lagrangian simulations of settling and agitated dense solid-liquid suspensions-achieving grid convergence. AIChE Journal, 64, 1147-1158.

Dionisi D. (2017). Biological wastewater treatment prcesses. CRC Press, Boca Raton.

Eggels, J. G. M. \& Somers, J.A. (1995). Numerical simulation of free convective flow using the latticeBoltzmann scheme. International Journal of Heat and Fluid Flow, 16, 357-364. 
Elimelech, M., Gregory, J., \& Jia, X. (2013). Particle deposition and aggregation: Measurement, modelling and simulation. Butterworth-Heinemann, Boston.

Feng, Z.G., \& Michaelides, E. (2009). Robust treatment of no-slip boundary condition and velocity updating for the lattice-Boltzmann simulation of particulate flows. Computers \& Fluids, 38, 370381.

Horii, K., Yamada, R., \& Harada, S. (2015). Strength deterioration of nonfractal particle aggregates in simple shear flow. Langmuir, 31, 7909-7918.

Kobayashi, M. (2004). Breakup and strength of polystyrene latex flocs subjected to a converging flow. Colloids and Surfaces A: Physicochemical and Engineering Aspects, 235, 73-78.

Kobayashi, M., Adachi, Y., \& Ooi, S. (1999). Breakup of fractal flocs in a turbulent flow. Langmuir, 15, 4351-4356.

Mesbah, A., Landlust, J., Huesman, A.E.M., Kramer, H.J.M., Jansens, P.J., \& Van den Hof, P.M.J. (2009). A model-based control framework for industrial batch crystallization processes. Chemical Engineering Research \& Design, 88, 1223-1233.

Mishra, P., \& Ein-Mozaffari, F. (2017). Using computational fluid dynamics to analyze the performance of the Maxblend impeller in solid-liquid mixing operations. International Journal of Multiphase Flow, 91, 194-207.

Moin, P. \& Mahesh, K. (1998). Direct numerical simulation: a tool in turbulence research. Annual Review of Fluid Mechanics, 30, 539-78.

Nagy, Z.K., Fevotte, G., Kramer, H., Simon, L.L. (2013). Recent advances in the monitoring, modelling and control of crystallization systems. Chemical Engineering Research \& Design, 91, 1903-1922.

Njobuenwu, D. O., \& Fairweather, M. (2017). Simulation of deterministic energy-balance particle agglomeration in turbulent liquid-solid flows. Physics of Fluids, 29(8), 083301.

Njobuenwu, D. O., \& Fairweather, M. (2018). Large eddy simulation of particle agglomeration with shear breakup in turbulent channel flow. Physics of Fluids, 30(6), 063303. 
Qi, N., Zhang, H., Zhang, K., Xu, G., \& Yang, Y. (2013). CFD simulation of particle suspension in a stirred tank. Particuology, 11, 317-326.

Reeks, M. W. (2014). Transport, mixing and agglomeration of particles in turbulent flows. Journal of Physics: Conference Series, 530, 012003.

Rubinstein, G.J., Derksen, J.J., Sundaresan, S. (2016). Lattice-Boltzmann simulations of low-Reynolds number flow past fluidized spheres: effect of Stokes number on drag force. Journal of Fluid Mechanics, 788, 576-601.

Sankaranarayanan, K., \& Sundaresan, S. (2008). Lattice Boltzmann simulation of two-fluid model equations. Industrial Engineering Chemistry Research. 47, 9165-9173.

Schiller, L., \& Naumann, A. (1933) Uber die grundlagenden Berechnungen bei der Schwerkraftaufbereitung. Ver. Deut. Ing. Z. 77, 318-320.

Seto, R., Botet, R., \& Briesen, H. (2011). Hydrodynamic stress on small colloidal aggregates in shear flow using Stokesian dynamics. Physical Review E, 84, 041405.

Spicer, P. T., Pratsinis, S. E., Raper, J., Amal, R., Bushell, G., \& Meesters, G. (1998). Effect of shear schedule on particle size, density, and structure during flocculation in stirred tanks. Powder Technology, 97, 26-34.

Succi, S. (2001). The lattice Boltzmann equation: For fluid dynamics and beyond. Oxford University Press, Oxford.

Sungkorn, R., \& Derksen, J.J. (2012). Simulations of dilute sedimenting suspensions at finite-particle reynolds numbers. Physics of Fluids, 24, 123303.

Tamburini, A., Brucato, A., Cipollina, A., Micale, G., \& Ciofalo, M. (2012). CFD predictions of sufficient suspension conditions in solid-liquid agitated tanks. International Journal of Nonlinear Sciences and Numerical Simulation, 13, 427-443. 
Tamburini, A., Cipollina, A., Micale, G., Brucato, A., Ciofalo, M. (2014). Influence of drag and turbulence modelling on CFD predictions of solid liquid suspensions in stirred vessels, Chemical Engineering Research and Design 92, 1045-1063.

Tourbin, M., \& Frances, C. (2009). Monitoring of the aggregation process of dense colloidal silica suspensions in a stirred tank by acoustic spectroscopy. Powder Technology, 190, 25-30.

Wen, C. Y., \& Yu, Y. H. (1966). Mechanics of fluidization. Chemical Engineering Progress Symposium Series, 62, 100-111.

Zhang, Y., Gao, Z., Li, Z., \& Derksen, J.J. (2017). Transitional flow in a Rushton turbine stirred tank. AIChE Journal, 63, 3610-3623. 
Table

Table 1. Definitions and settings of dimensionless numbers

\begin{tabular}{|l|l|}
\hline $\operatorname{Re}=N D^{2} / \nu$ & $4000,6000,8000$ \\
\hline$\langle\phi\rangle=n \frac{\pi}{6} d^{3} / T^{3}$ & 0.0983 \\
\hline$d / T$ & 0.00909 \\
\hline$\rho_{s} / \rho$ & 2.23 \\
\hline$\theta=\rho N^{2} D^{2} /\left[g d\left(\rho_{s}-\rho\right)\right]$ & 260 \\
\hline$\beta_{a}=\beta /\left(g \rho_{s} \frac{\pi}{6} d^{3}\right)$ & $0.10-27$ \\
\hline$\delta_{a}=\delta_{0} / d$ & $0.30,0.40,0.50$ \\
\hline
\end{tabular}


Figures

Figure 1. (a) Top view of the mixing tank. (b) Side view of the mixing tank. The pitched-blade impeller rotates such that it pumps in the negative $z$ direction. The thickness of the impeller blades is $t_{b}=0.021 D$. The origin of the Cartesian coordinate system is in the center of the bottom wall.

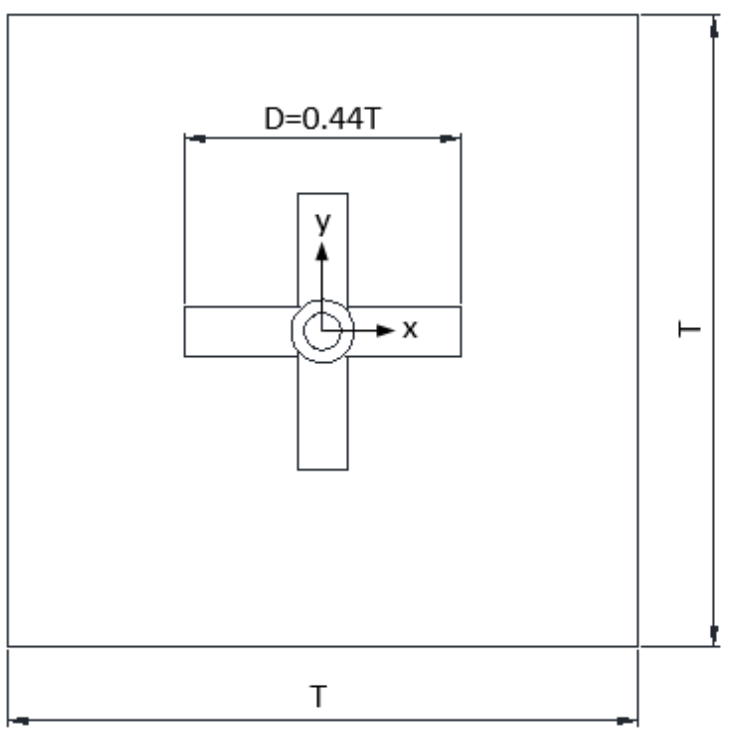

(a)

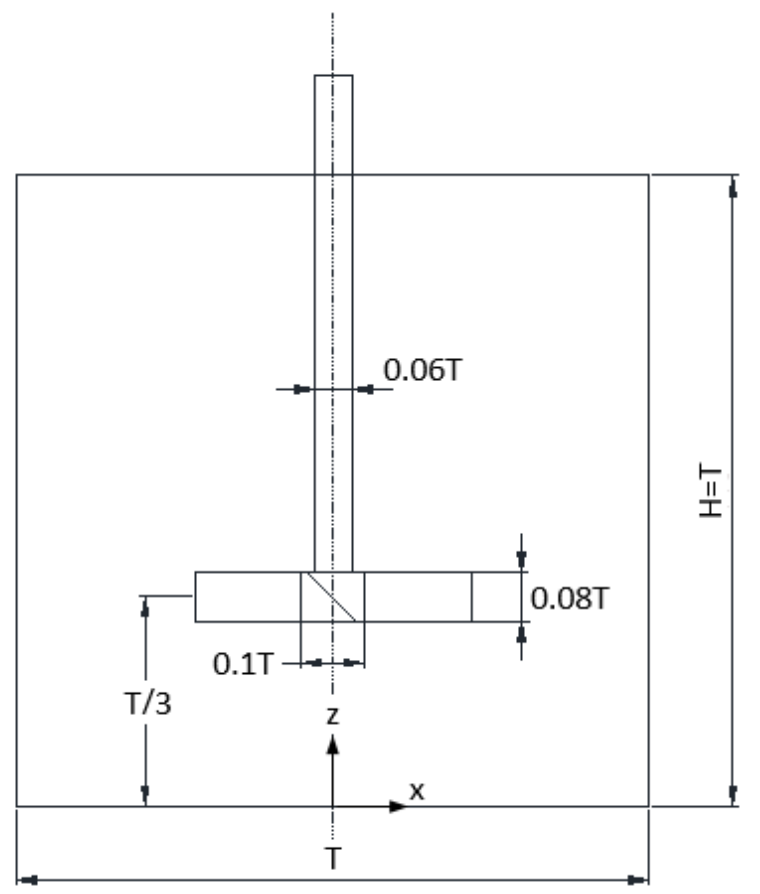

(b) 
Figure 2. Instantaneous realizations of particles in a $y z$ slice with thickness $d$ through the center of the tank for the non-aggregating simulation under base-case conditions that starts with particles forming a dense layer on the tank bottom at moments $t N=1,4,16$ and 64 (a to d) respectively.

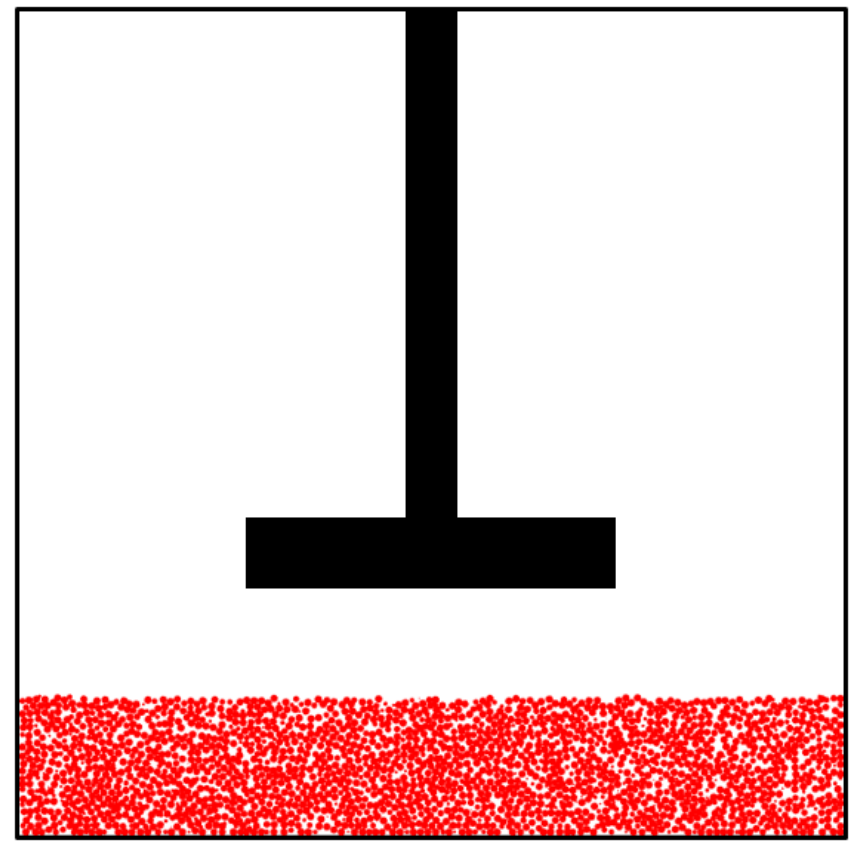

(a)

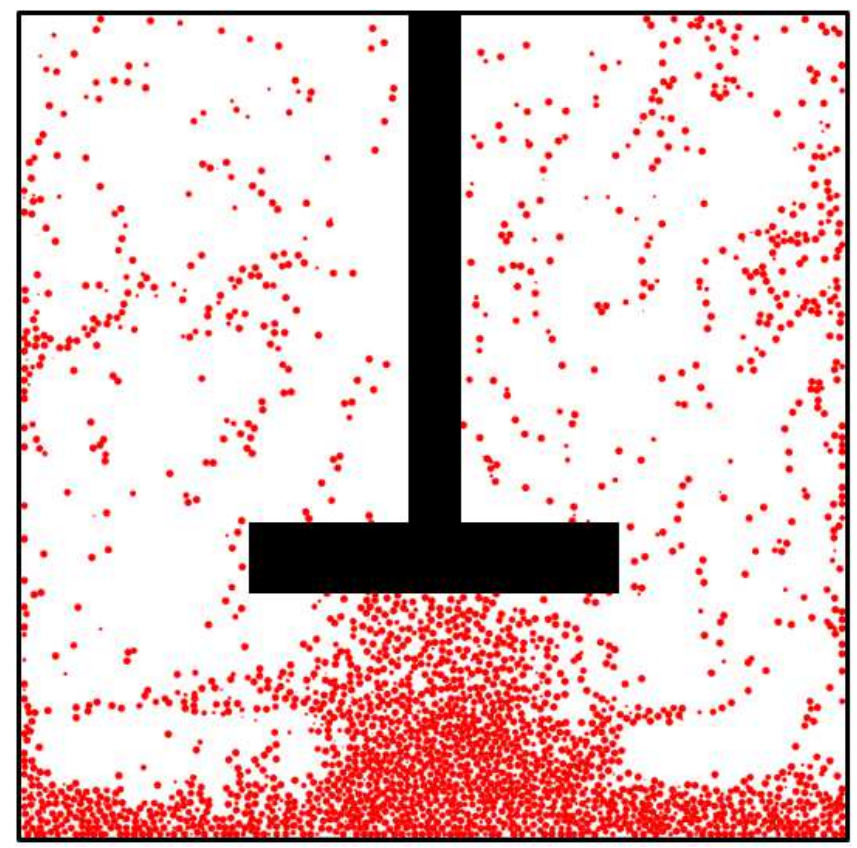

(c)

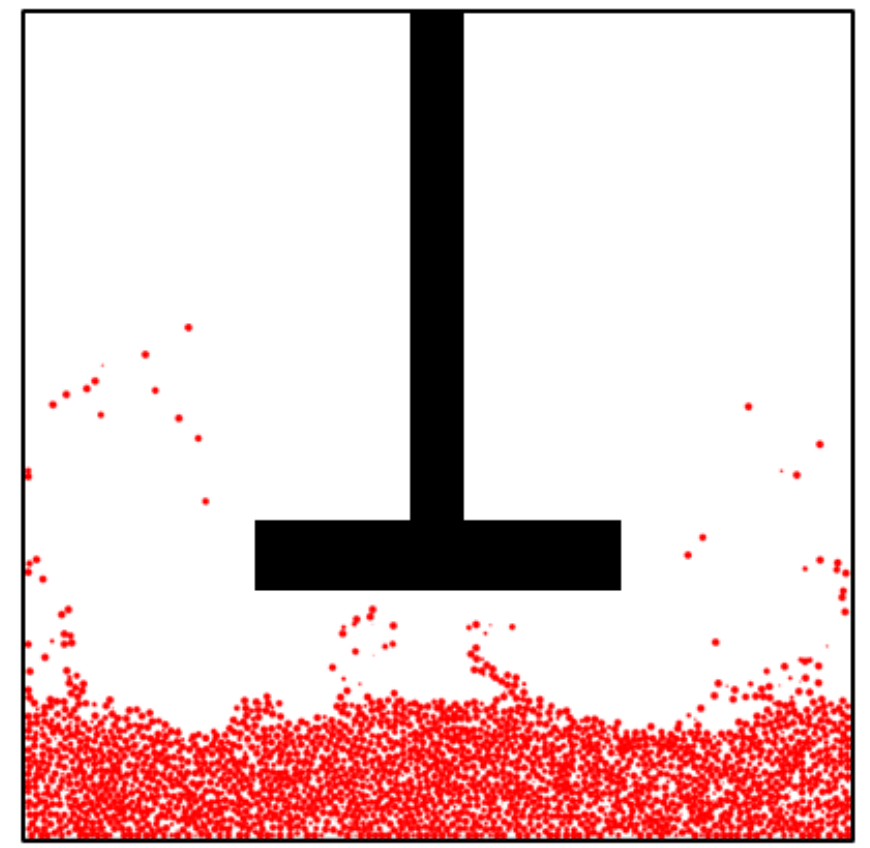

(b)

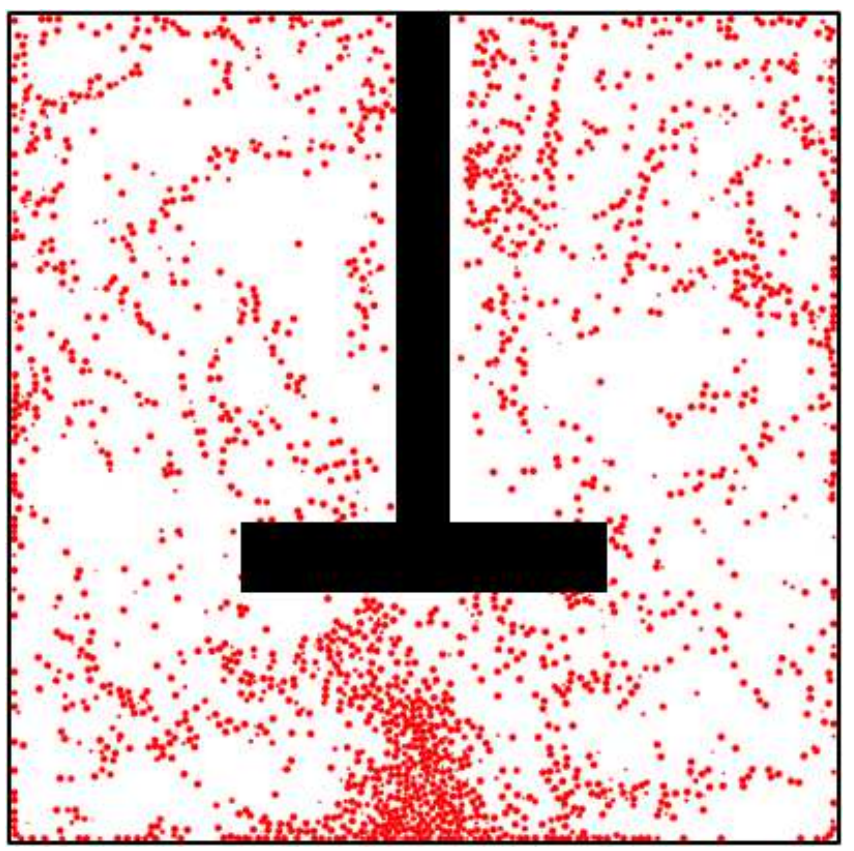

(d) 
Figure 3. Snapshots of liquid velocity vectors in the $y z$ plane through the center of the tank for the basecase non-aggregating simulation at moments $t N=1,4,16$ and 64 (a to d) respectively.

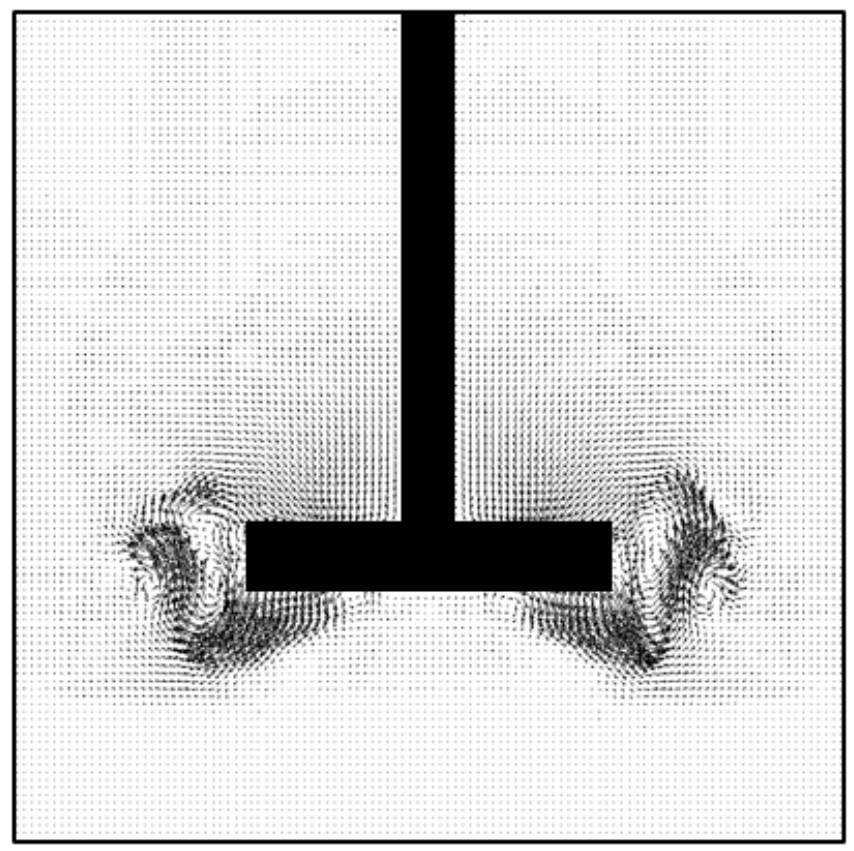

(a)

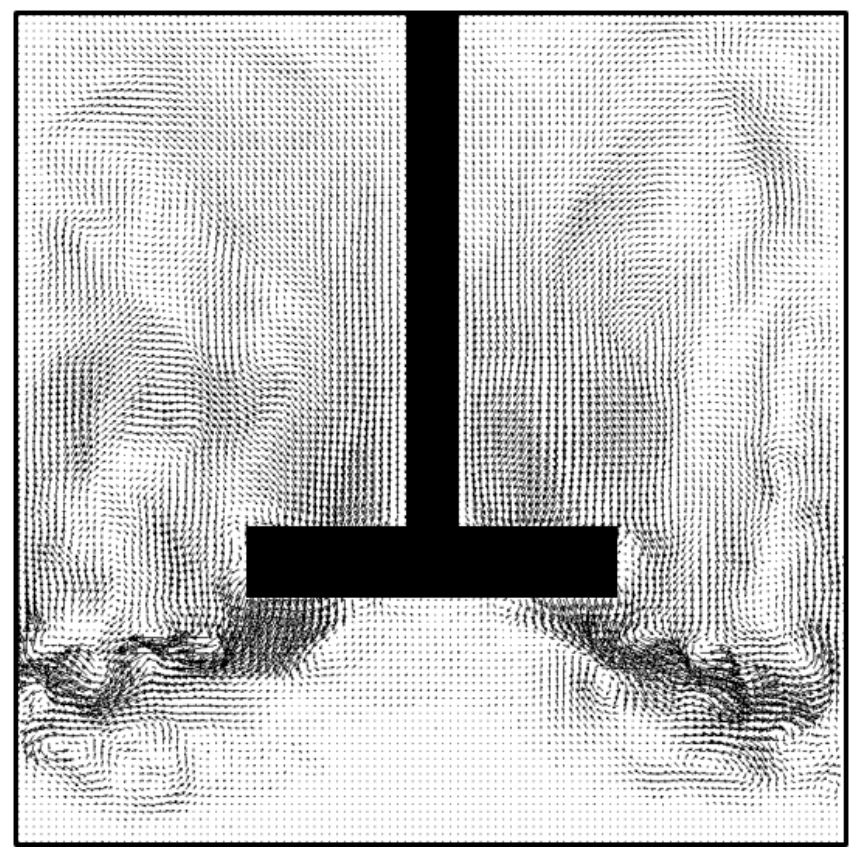

(c)

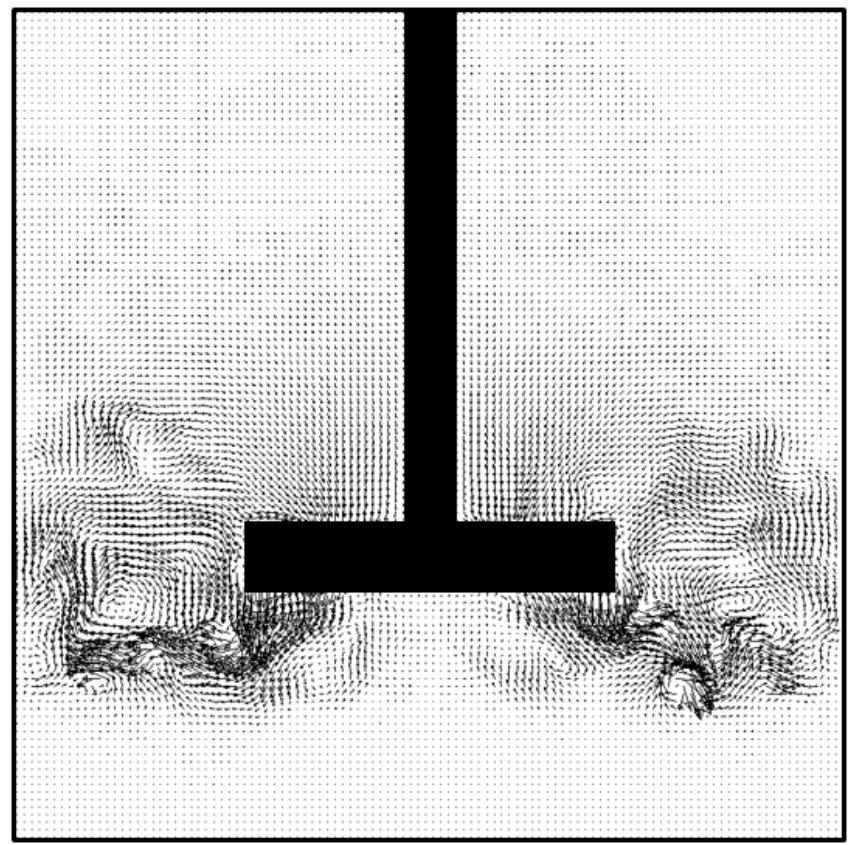

(b)

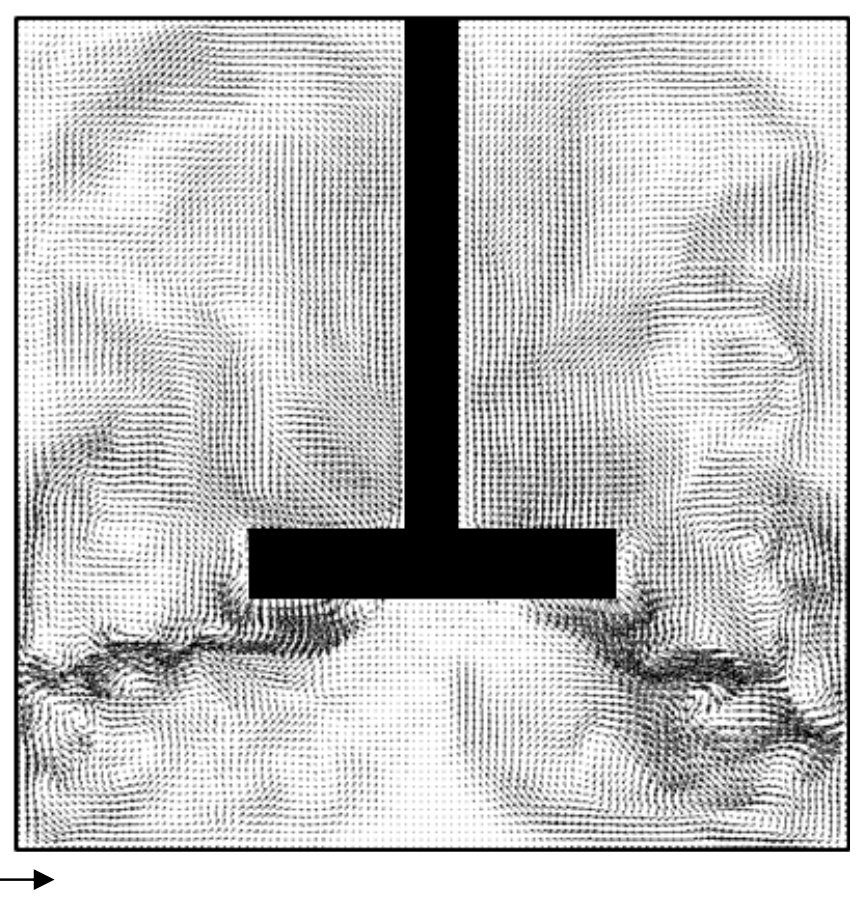

(d) 
Figure 4. Time-averaged (over the time window $90 \leq t N \leq 100$ ) solids volume fraction contours in a vertical plane through the center of the mixing tank of the base-case non-aggregating system.

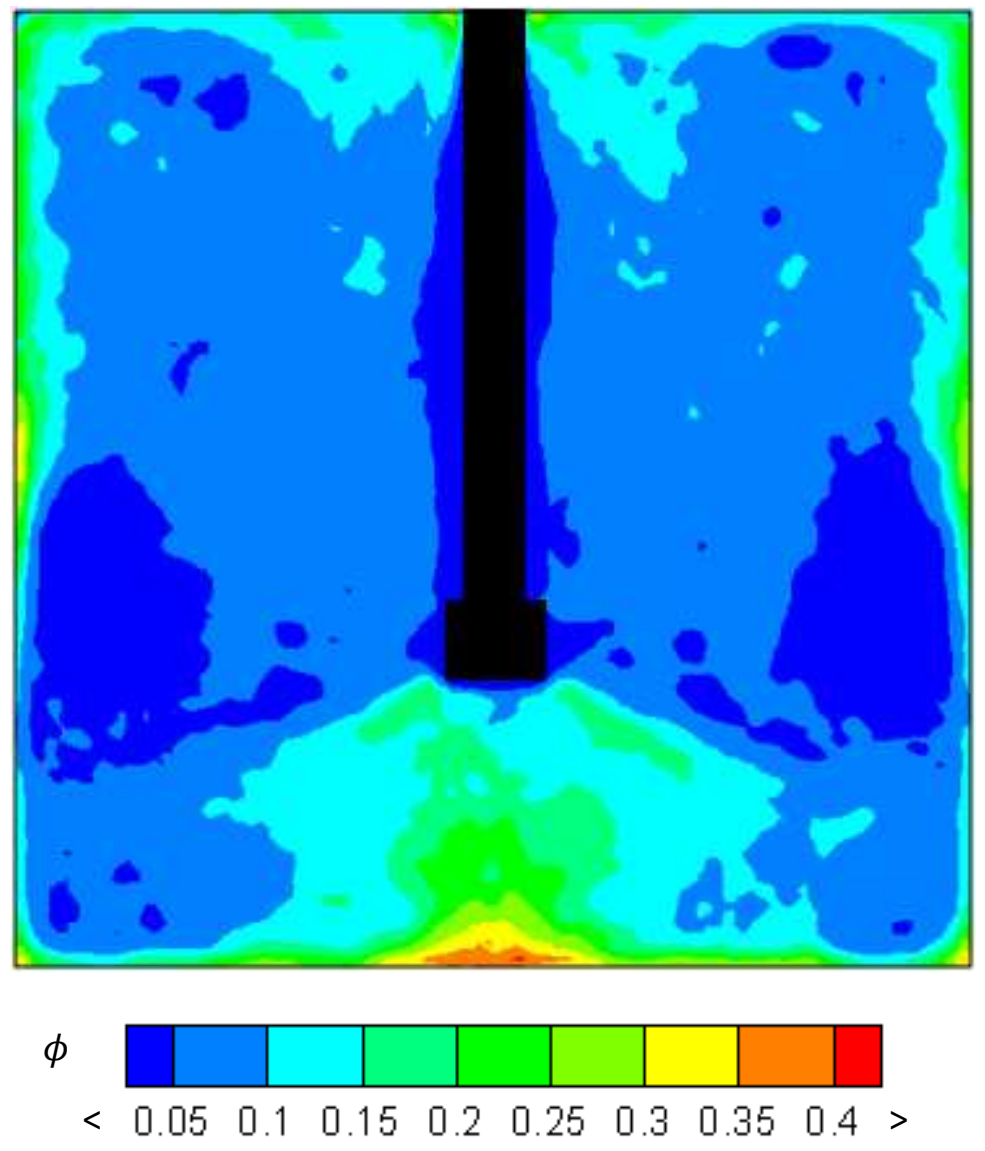


Figure 5. Average coordination number $C_{\text {avg }}$ as a function of time for the base-case non-aggregating system starting from a dense layer of particles on the bottom.

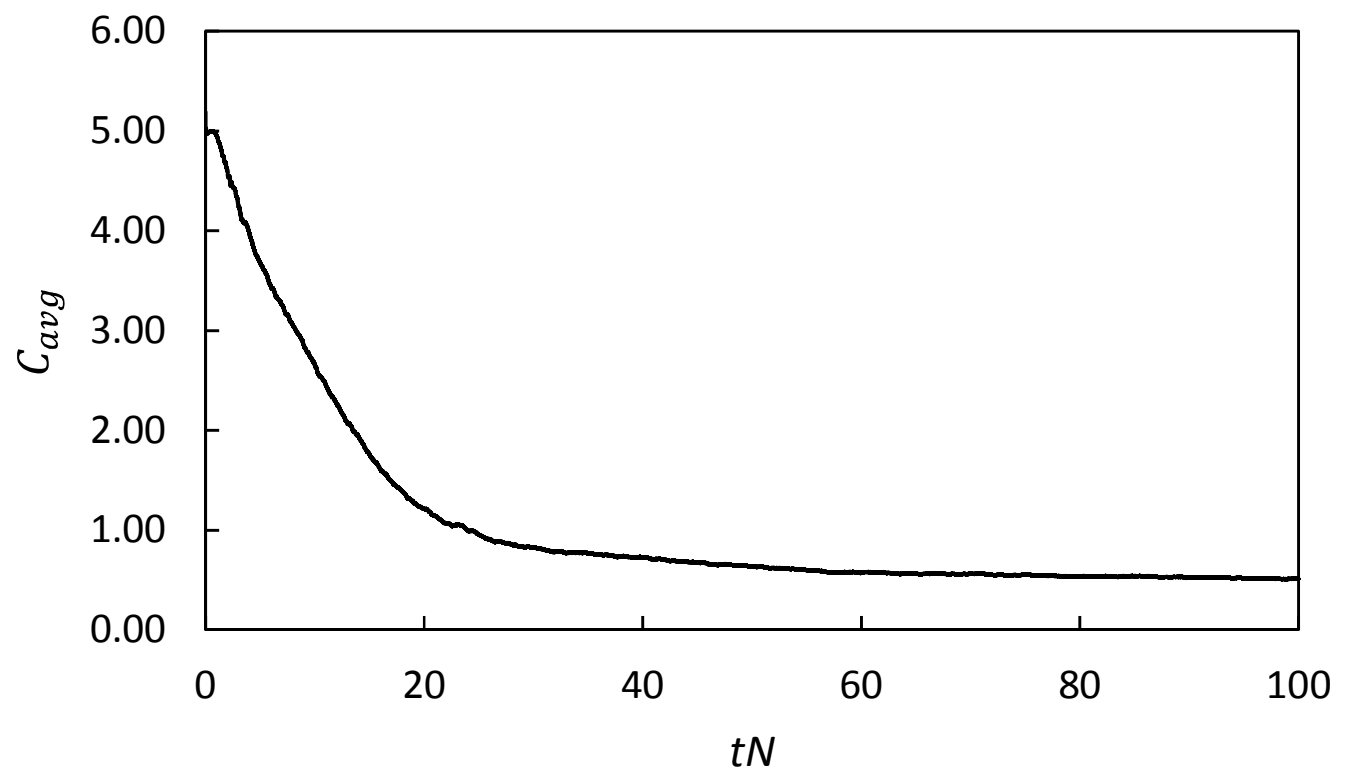


Figure 6. Average coordination number $C_{a v g}$ as a function of time for aggregating systems over a range of dimensionless interaction strengths $\beta_{a}$ (as indicated) for dimensionless interaction distance $\delta_{a}=0.4$ and further base-case conditions.

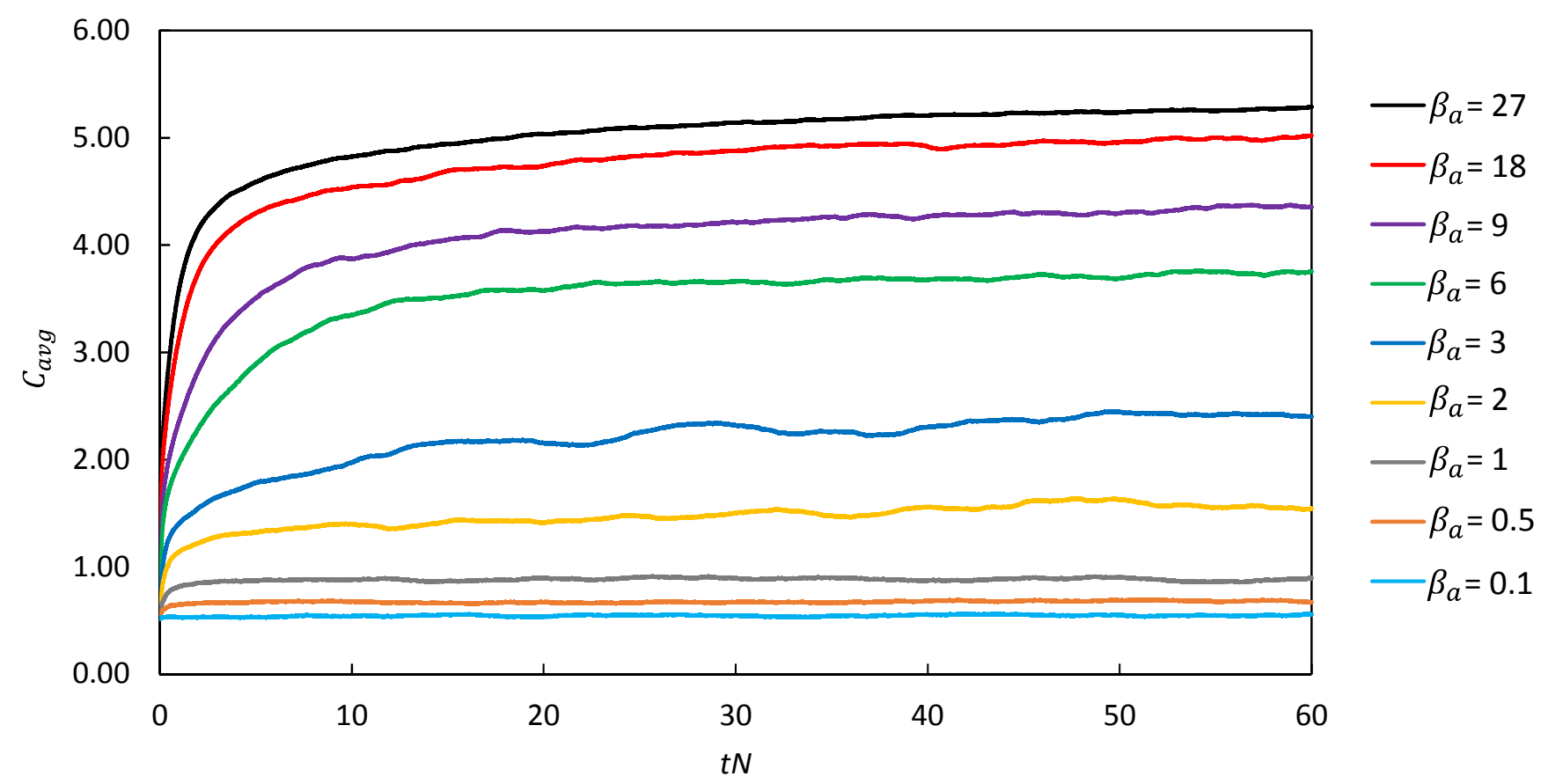


Figure 7. Instantaneous realizations of particles in a $y z$ slice with thickness $d$ through the center of the tank at $\beta_{a}=9$ and $\delta_{a}=0.4$ : (a) $t N=1$; (b) $t N=4$; (c) $t N=16$ and (d) $t N=60$. Color coding: blue: single particle; cyan: a doublet; magenta: a triplet; red a quartet; green: an aggregate of 5 or more particles.

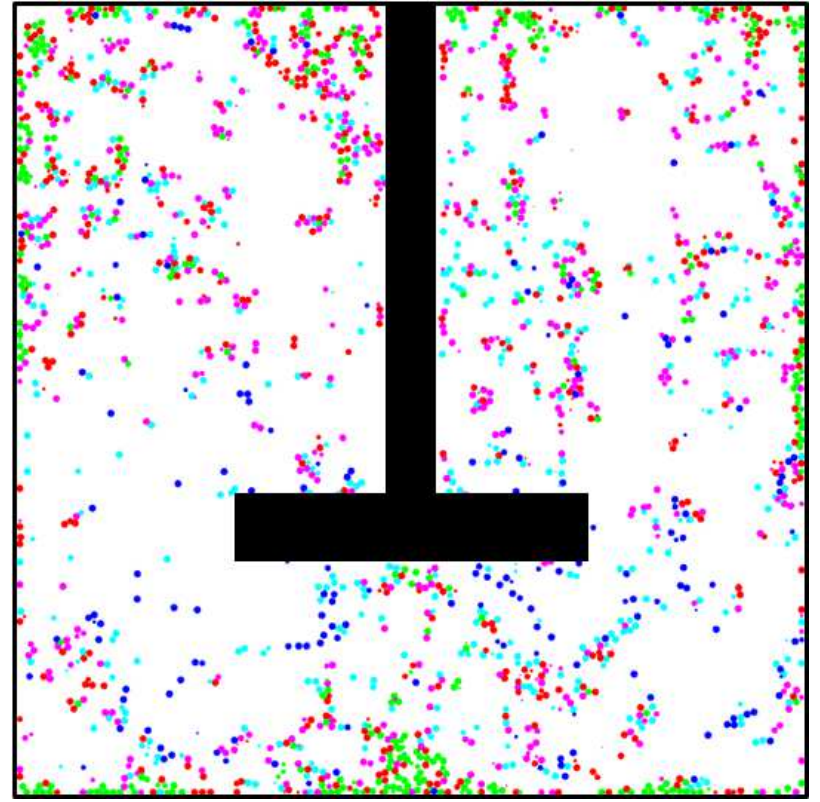

(a)

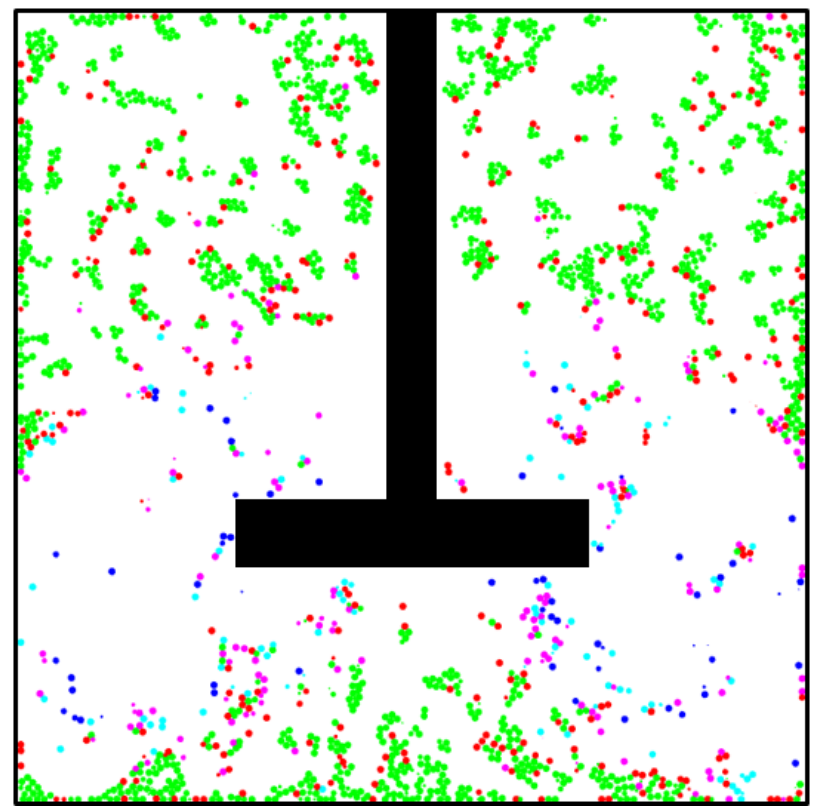

(c)

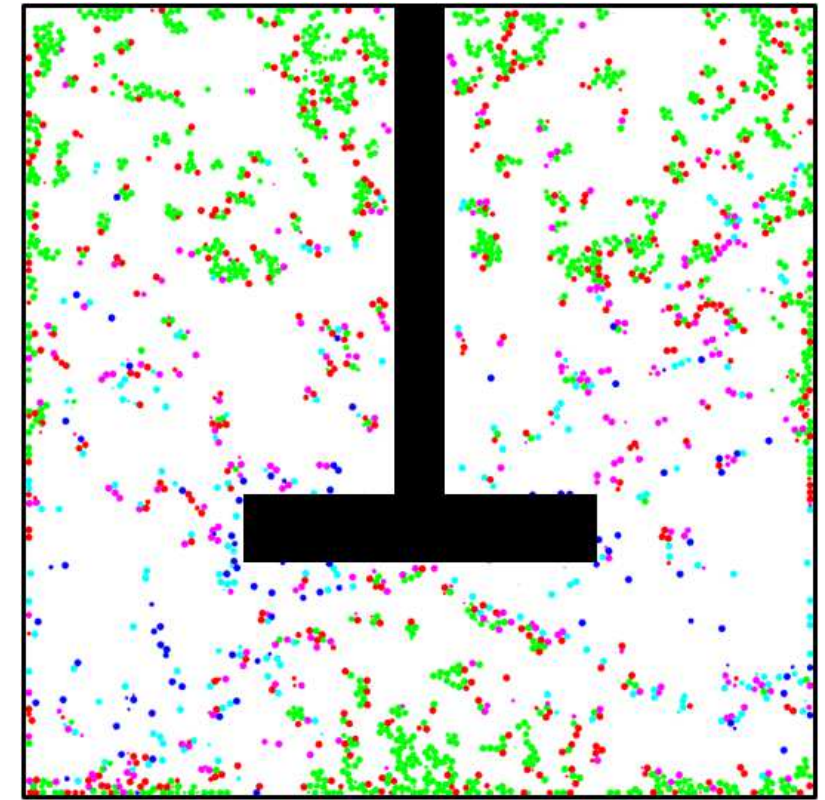

(b)

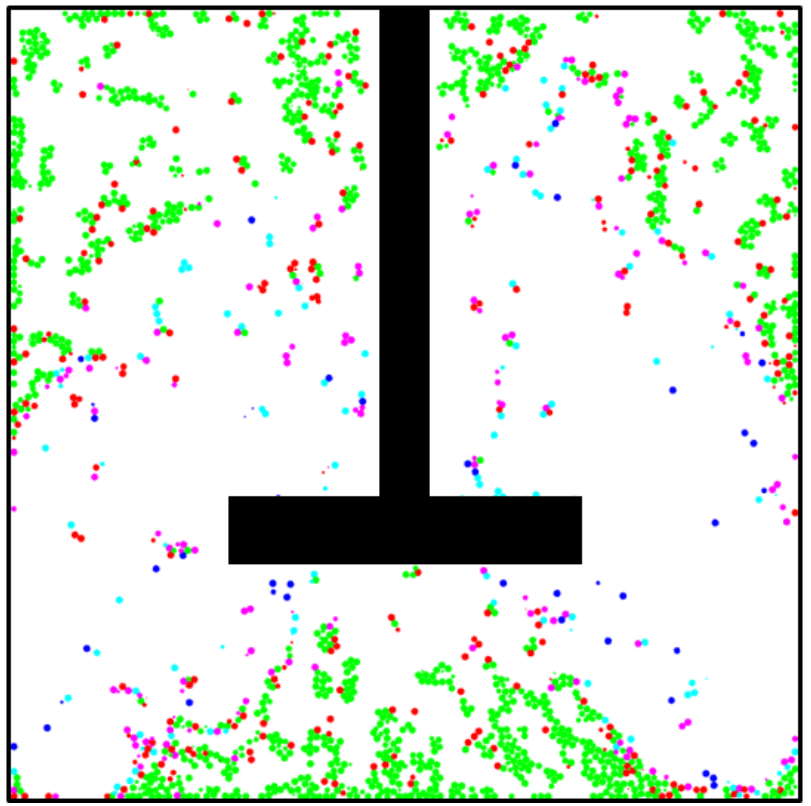

(d) 
Figure 8. Time-averaged (over $50 \leq t N \leq 60$ ) solids volume fraction contours in a vertical plane through the center of the mixing tank for different strength of aggregation: a) $\beta_{a}=1$, (b) $\beta_{a}=3$, (c) $\beta_{a}=9$. $\operatorname{Re}=$ 4000 and $\delta_{a}=0.4$.

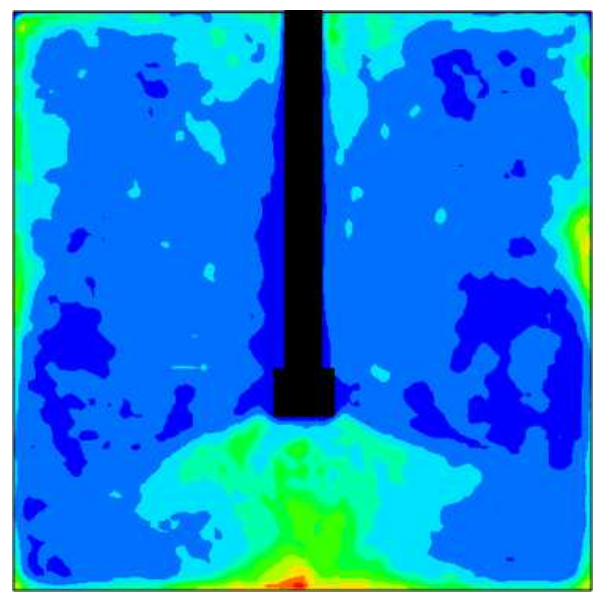

(a)

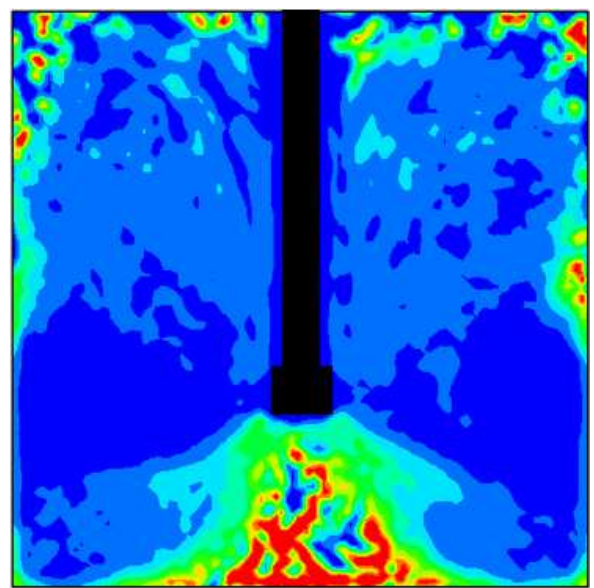

(b)

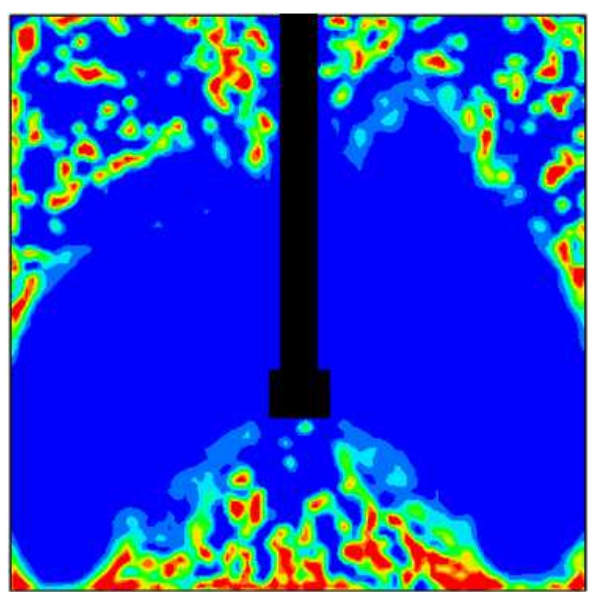

(c)

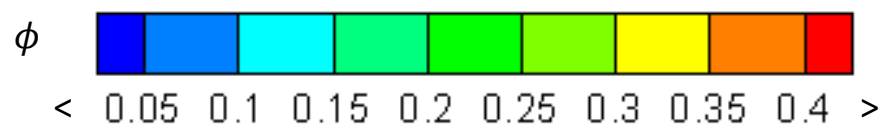


Figure 9. Steady-state average coordination number $C_{a v g}$ as a function of the aggregation force parameters $\beta_{a}$ and $\delta_{a}$.

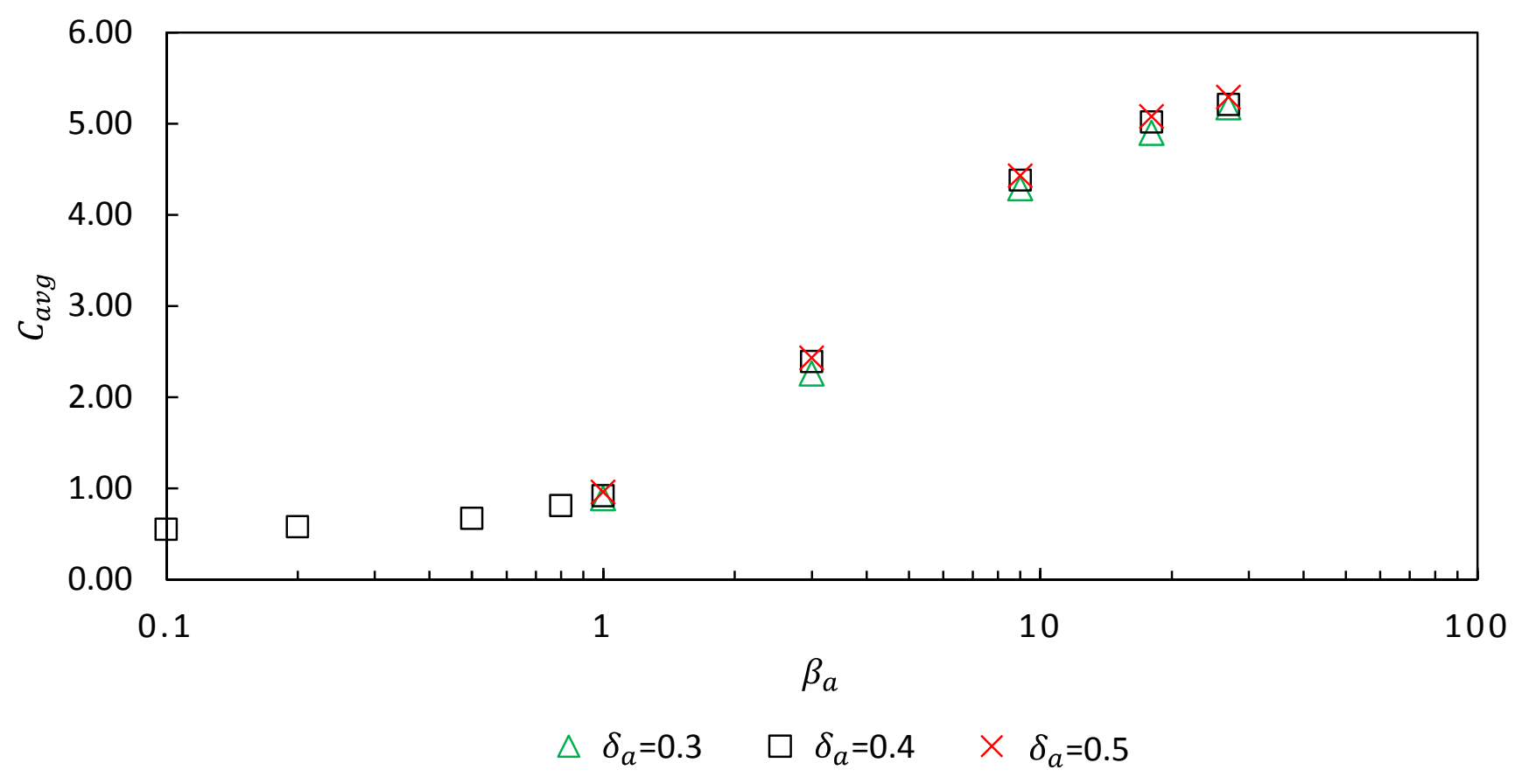


Figure 10. Time-series of $C_{a v g}$ for $\operatorname{Re}=4000,6000$ and 8000 with $\beta_{a}=3$ and $\delta_{a}=0.4$.

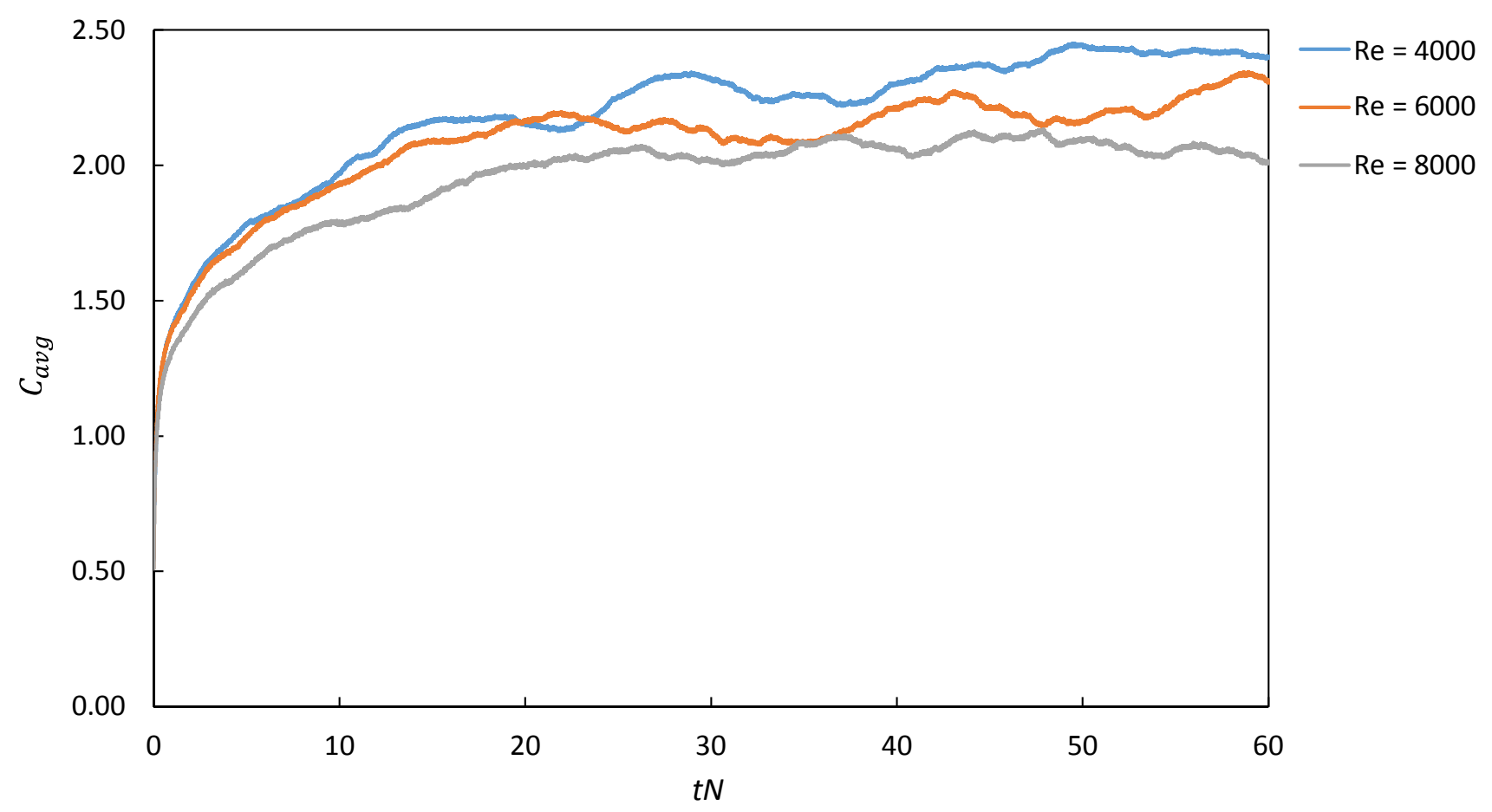


Figure 11. Time-averaged turbulent kinetic energy (normalized by the square of the impeller tip speed) in the vertical $y z$ plane through the center of the tank. Averaging over 10 impeller revolutions $(50 \leq t N \leq$ 60). From left to right: $\operatorname{Re}=4000,6000$ and $8000 ; \beta_{a}=3$ and $\delta_{a}=0.4$.

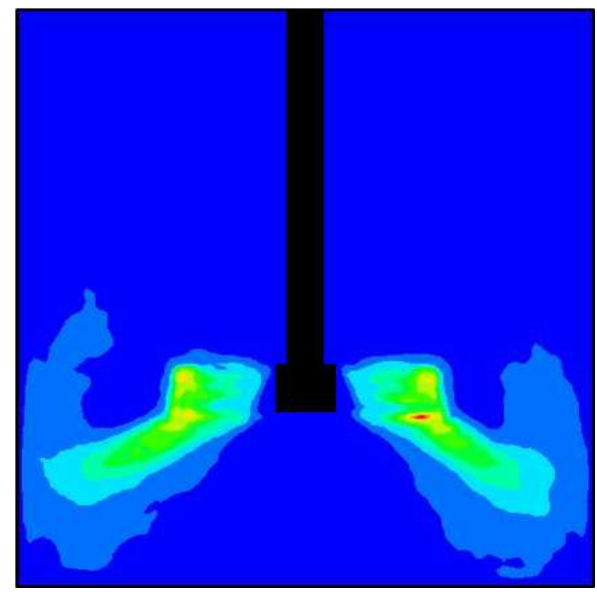

(a)

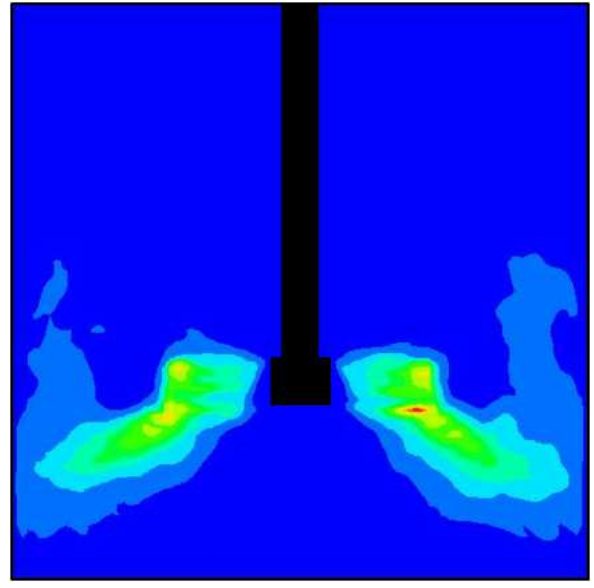

(b)

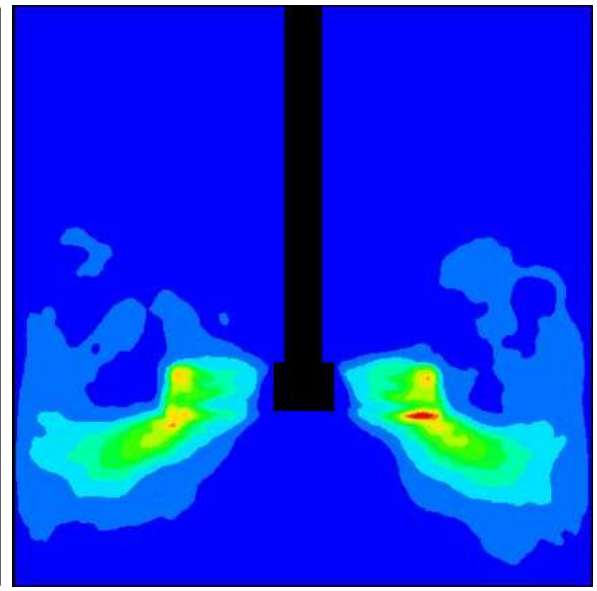

(c)

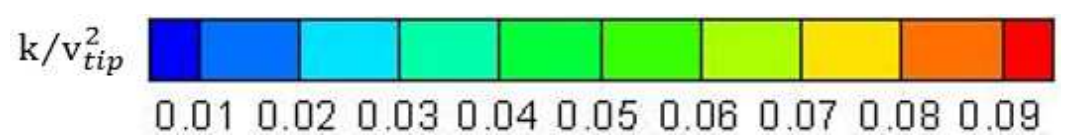


Figure 12. Aggregate size distributions, averaged over the time-interval $50 \leq t N \leq 60$, with $\operatorname{Re}=4000$, $\delta_{a}=0.4$ and a range of aggregation strengths $\beta_{a}$. The symbol $\bar{\psi}$ indicates the volume of solids contained in each size class divided by the total volume of solids. (a) $\beta_{a}$ from $0-1$; (b) $\beta_{a}=3$.

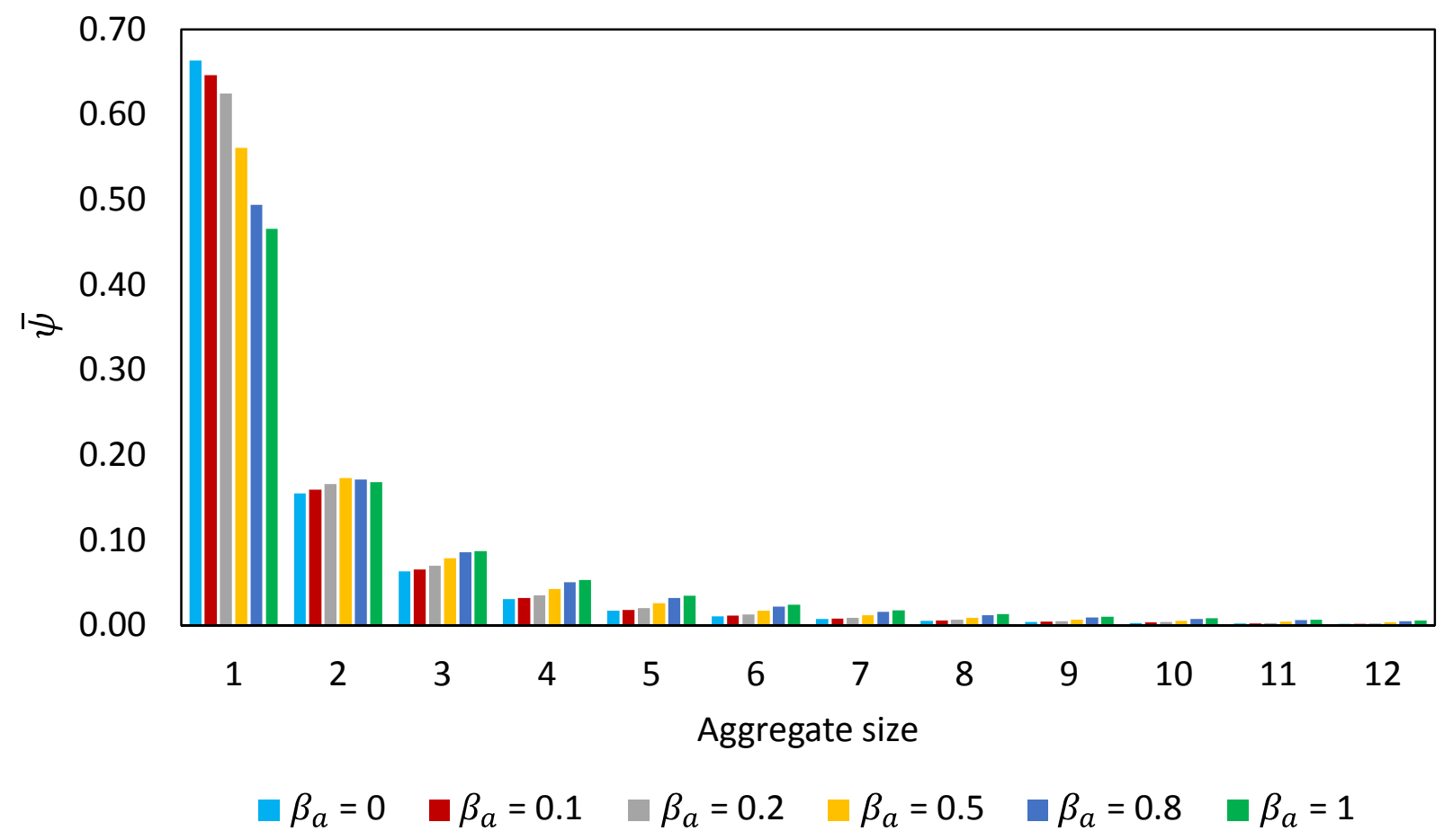

(a)

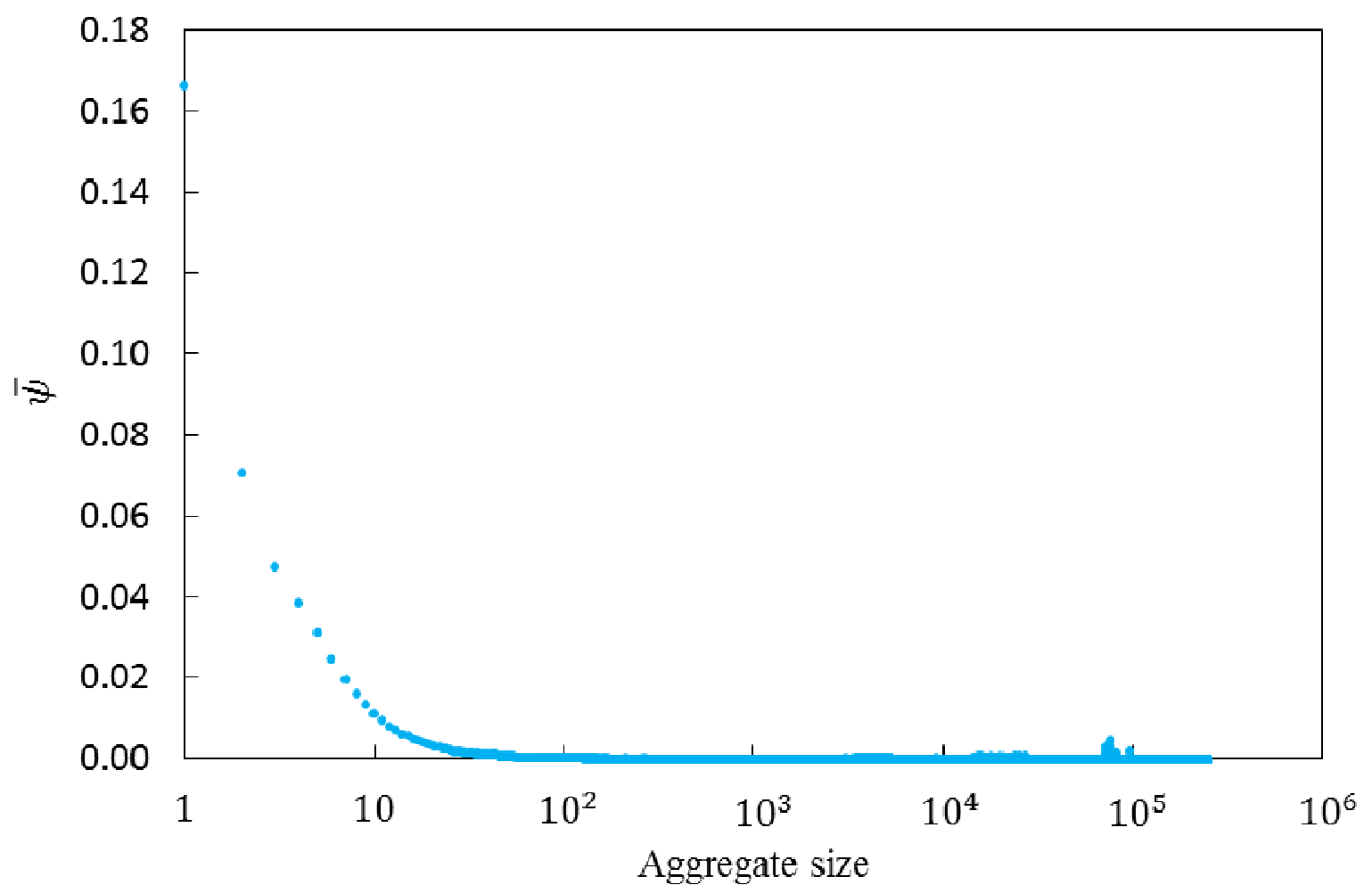

(b) 